\title{
PROBLEMAS RELATIVOS A LA PRUEBA EN CADA ETAPA DE LA ACTIVIDAD PROBATORIA EN EL PROCEDIMIENTO DE FAMILIA
}

\author{
PROBLEMS RELATED TO EVIDENCE ON EACH ONE OF THE \\ MOMENTS OF EVIDENTIARY ACTIVITY IN FAMILY LAW \\ PROCEEDING
}

\author{
Jesús EZURMENDia ÁlVAREZ
}

\begin{abstract}
RESUMEN: En el presente artículo se pretende trasladar la discusión sobre los momentos de la actividad probatoria, y los problemas que cada uno de ellos engendra, al derecho procesal de familia, específicamente al procedimiento reformado contenido en la Ley No 19.968. Dada su estructura y principios rectores, es posible reconocer claramente los momentos de inclusión, valoración y eventual aplicación de un estándar de prueba, pudiendo evidenciar en cada uno de ellos determinados problemas o peligros derivados de la actividad corroborativa de las partes y el juez. Este trabajo pretende presentar y describir, sin fines exhaustivos, algunos de los mencionados problemas, haciéndose cargo de la insatisfactoria aplicación de la teoría de la prueba al proceso de familia a la luz del aumento de facultades entregadas a los tribunales de familia en época reciente.
\end{abstract}

Palabras clave: Derecho Procesal Civil, Derecho Probatorio, Derecho de Familia, Derecho Procesal de familia.

ABSTRACT: This article aims to draw attention to the discussion regarding the moments of evidentiary activity, and the problems that each one of those steps could engender, to family law process, specifically to the reformed proceedings enshrined in the Act No 19.968. In accordance with its structure and ruling principles, it is possible to acknowledge the moments of conformation, evaluation and the application of a standard of proof, evidencing the problems and risks attached to every stage of the corroborative activity performed by parties and adjudicators. This work expects to present and describe, with no exhaustive ambition, some of the aforementioned issues, taking charge of the unsatisfactory application of evidence law to family proceedings given the recent increase of powers entrusted to Family Courts.

Key words: Civil Process, Evidence Law, Family Law, Family Law Proceedings.

\section{LAS ETAPAS DE LA ACTIVIDAD PROBATORIA Y LOS PROCEDIMIENTOS DE FAMILIA}

En general los procedimientos reformados, y en especial aquellos regulados por la Ley No 19.968 sobre Tribunales de Familia ${ }^{1}$, permiten distinguir en la estructura de sus

\footnotetext{
* Magister en Derecho (LLM) University College of London. Profesor Asistente Departamento Derecho Procesal Universidad de Chile. Dirección postal. Av. Santa María 076, quinto piso, Providencia, Chile. Dirección electrónica: jezurmendia@derecho.uchile.cl.

1 En adelante "LTF".
} 
diversos periodos de tramitación de forma sustancialmente más clara aquello que parte importante de la literatura dedicada a la teoría de la prueba ha denominado "las etapas de la actividad probatoria" en perspectiva a su contraparte $-\mathrm{y}$ supletoria- regulación procesal civil $^{2}$. Estas etapas, han sido señaladas por voces autorizadas como Ferrer ${ }^{3}$ y Taruffo ${ }^{4}$, en derecho continental y, con matices, por Murphy ${ }^{5}$, Roberts ${ }^{6}$ y Stein ${ }^{7}$ en el derecho anglosajón, como estadios tripartitos de actividad relativa a la evidencia, consecutivos, correlativos y consecuenciales entre sí, en la que intervienen tanto las partes como tribunal, y que impactan en lo que, al final del proceso, ha de tenerse por probado (en términos de Ferrer "que esté probado que $\mathrm{p}^{\prime 8}$ ) aludiendo a la acepción de la polisemia de la palabra prueba, en este caso, como resultado. Los mencionados momentos de actividad probatoria han sido diagramados, aunque con breves matices, como aquellos correspondientes a, en primer lugar, a) la etapa de conformación del material probatorio, también llamado período de inclusión o incorporación de la prueba; b) etapa o momento de valoración de la prueba; y c) momento de aplicación de un estándar de prueba9.

Esta estructura secuencial de la actividad referida a los hechos estaría presente en el proceso de corroboración o averiguación sobre la veracidad de dichos enunciados fácticos, independiente de la claridad con la que pueda aparecer manifestada en la consagración normativa de leyes y códigos procesales, existiendo a tal respecto regulaciones en las que la distinción antedicha es difusa y casi ininteligible -como los códigos procesales de comienzos del siglo XX- y, por otro lado, estatutos de data reciente en las que es posible diagramar en forma específica cada uno de estos momentos, siendo, como se ha mencionado al comienzo, la Ley No 19.968 uno de los más claros ejemplos de esto.

Así, los momentos de conformación del material probatorio, valoración y aplicación de un estándar de prueba son claramente diferenciables en la estructura del Procedimiento Ordinario ante los jueces de Familia ${ }^{10}$, y en general en la mayoría de los procedimientos especiales de la Ley, sin embargo, esta distinción relativamente instalada y clara en la literatura probatoria en ambos lados del Atlántico ${ }^{11}$, que ha sido tratada en forma lata con ocasión del proceso penal ${ }^{12}$, no ha sido absorbida ni particularmente revisada con ocasión del proceso de familia tanto a nivel de especialistas teóricos en el área, como a nivel de operadores del sistema ${ }^{13}$. El objetivo de este trabajo, en atención a su extensión, es visibilizar algunos de los problemas que desde la teoría de la prueba se pueden apreciar en la LTF en cada una

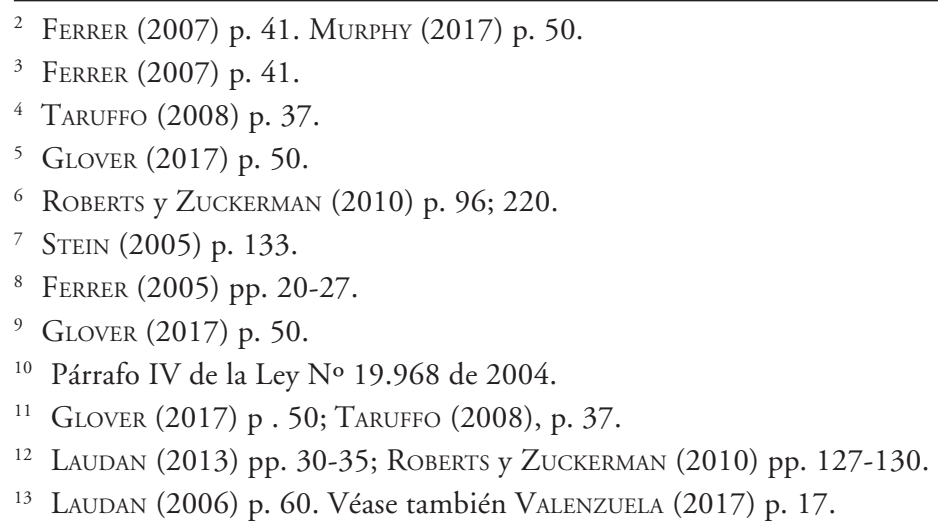


de las precitadas etapas de la actividad referida a la prueba, haciendo presente que, sin fines exhaustivos, se apunta a evidenciar cuestiones relevantes a este respecto, planteando tanto la discusión como algunas sugerencias.

\section{ETAPA DE CONFORMACIÓN DEL MATERIAL PROBATORIO, EN ESPECIAL LA EXCLUSIÓN DE PRUEBA}

Se entiende como etapa de conformación del material probatorio aquel momento del proceso en que la evidencia que posteriormente deberá ser valorada por el sentenciador es incorporada y depurada conforme a ciertos elementos, establecidos normativamente, y que dicen relación con criterios de admisibilidad y relevancia ${ }^{14}$. En general, se ha señalado que los sistemas que consagran como principio formativo la libertad de prueba, tal como ocurre en la LTF Art. 28, tienden a entregar al sentenciador una fórmula que permita disponer de toda la información disponible para la toma de decisión, entendiendo que entre más y mejor sea la prueba que recaiga sobre los enunciados fácticos propuestos por las partes, más fiable debería ser, en teoría, la decisión probatoria a tal respecto. De esta manera, se eliminan "barreras de entrada" a través de imposiciones legislativas que limiten la posibilidad de probar en juicio los hechos a través de determinados medios de prueba o, contrafaz de moneda, que obliguen a probar ciertas circunstancias a través de medios específicos. Asimismo, se eliminan inhabilidades como las tachas en materia testimonial y la distinción entre la naturaleza del documento para su incorporación al proceso. En ese sentido, se dice entonces, que la prueba libre busca que toda la evidencia disponible sea allegada al proceso, de forma tal de formar un acervo probatorio lo más completo posible, para que, epistémicamente se cuente con el mayor grado de conocimiento respecto de la eventual veracidad de las alegaciones de las partes.

Sin embargo, dentro de la etapa de conformación/inclusión se verifica un segundo proceso, inherente a la etapa en comento, el de exclusión de prueba. No obstante, lo contradictorio que pueda parecer, en el contexto judicial la prueba está regida por un contexto institucional ${ }^{15}$, que obliga a que la prueba que pueda valorarse al momento de la decisión sea solo aquella que está de acuerdo con ciertos parámetros establecidos por la ley, y que muchas veces restringen o limitan la libertad de prueba ${ }^{16}$. Se trata, en consecuencia, del establecimiento de reglas contra epistémicas, que atentan contra el acceso a más información para la toma de decisión ${ }^{17}$, pero que la realidad del proceso y los intereses que deben ponderarse y protegerse obligan a respetar al momento de decidir que evidencia debe ser admitida.

Dentro de este conjunto de reglas de exclusión suelen presentarse mecanismos de filtrado que permiten descartar la prueba que no será de utilidad para el proceso, o que no debería ser considerada por ser vulneratoria de los derechos de alguna de las partes. A tal respecto, en lo pertinente a la LTF toma particular relevancia lo dispuesto en el artículo

\footnotetext{
14 TARuffo (2008) pp. 37-38.

15 BayOn (2010) p. 7. También Véase GASCón (2005) p. 128.

16 En el caso de la LTF dicha regulación se establece en el artículo 31.

17 Bayon (2010) p. 7. Usando la voz extra epistémicos véase también Bentham (2001) p. 391.
} 
EZURMENDia Álvarez, Jesús — "Problemas relativos a la prueba en cada etapa de la actividad probatoria ..."

31, el que establece un sistema de exclusión de prueba fundado en motivos de pertinencia, notoriedad de los hechos, sobreabundancia y, especialmente, aquellas que hubieren sido obtenidas con infracción de garantías fundamentales ${ }^{18}$. Esta depuración probatoria tiene lugar, por regla general, en la audiencia preparatoria, conforme lo dispuesto en los artículos 31 y 61 No 8 LTA y constituye la oportunidad procesal en la cual se fijan, junto con los hechos a probar en la audiencia de juicio, las piezas de evidencia con las que contará en la audiencia de juicio el tribunal para decidir el asunto. La norma, cabe señalar, expresa que las exclusiones son una excepción y que, salvo alguno de los supuestos en ella descrita, el resto de la prueba deberá ser admitida. Al respecto, y para los fines de este ensayo, las reglas que más llaman la atención son las referidas a la prueba ilícita ${ }^{19}$, en el sentido de examinar cuales son los criterios que podrían seguirse, en abstracto, para entender que ha existido una garantía fundamental conculcada que devenga en la exclusión de prueba, y por otro lado, cuál ha sido la aproximación de la judicatura de familia en la utilización de los mecanismos de exclusión, teniendo presente, por ejemplo, que el desarrollo de la eficacia procesal de los derechos humanos, como fundamento de la exclusión, tiene data reciente y ha ido de la mano del proceso penal reformado ${ }^{20}$, y de desarrollos relativamente actuales en materia debido proceso y garantías procesales. Asimismo, la ilicitud de la prueba obtenida por sujetos particulares ha sido poco tratada en nuestra literatura, y no participa de las mismas aproximaciones en la doctrina ha dado a la exclusión de evidencia obtenida por agentes estatales dotados de facultades de investigación ${ }^{21}$.

Como bien señala Jara, la construcción de un sistema de exclusión de prueba basada en la aplicación de principios constitucionales a un proceso de derecho privado resulta compleja ${ }^{22}$. Especialmente, teniendo presente la naturaleza de los litigios de la judicatura de familia, es del todo esperable que exista una relación de cercanía entre los litigantes que presente o posicione a gran parte de la evidencia disponible en el límite de la vulneración de la privacidad, la honra, la inviolabilidad del hogar, etc. de un sujeto, pudiendo caer en la tentación de la sobre-exclusión ${ }^{23}$. Lo anterior, creemos, es particularmente relevante en relaciones procesales que tienen, además, un correlato en relaciones de parentesco o familia,

\footnotetext{
18 Art. 31: El juez de familia, luego de estudiar la admisibilidad de las pruebas ofrecidas, de resolver las convenciones probatorias y de escuchar a las partes que hubieren comparecido a la audiencia preparatoria, ordenará fundadamente que se excluyan de ser rendidas en el juicio aquellas que fueren manifiestamente impertinentes, tuvieren por objeto acreditar hechos públicos y notorios, resulten sobreabundantes o hayan sido obtenidas con infracción de garantías fundamentales.

19 Los criterios de prueba impertinente, basada en hechos públicos o notorios y sobreabundantes no suelen generar problemas de interpretación. En caso de consulta puede revisarse la casuística en: $A$. con S. (2015); BETANZO con FUENTES (2011)."

20 Jara y Jara (2014).

${ }^{21}$ En ese sentido destaca el trabajo de Correa Zacarías, en el que se analiza el estado de la doctrina procesal penal chilena respecto a la exclusión de prueba obtenida por particulares, diferenciando entre prueba de cargo y de descargo. CORREA (2016) pp. 118-125.

22 Jara y Jara (2014).

${ }^{23}$ Siguiendo los ejemplos dados por Jara, podría pensarse en casos de divorcio sanción por causales del art. 54 de la Ley de Matrimonio Civil, especialmente la infidelidad reiterada $\left(2^{\circ}\right)$, conducta homosexual $\left(4^{\circ}\right)$. JARA y JARA (2014).
} 
en la que el acceso a la información privada del otro puede ser difícil de determinar (entendido por ejemplo entre cónyuges o entre padres e hijos).

A nivel jurisprudencial, dado el momento procesal en el que se verifica la exclusión, no es abundante la jurisprudencia contrastable a este respecto, sin perjuicio de poder encontrar a aproximaciones interesantes en determinados casos que llegan a la Corte por la vía de impugnación. Así, en una causa de medidas de protección, la denunciante y madre del menor recurre de queja en contra del juez de familia quien excluyó una prueba audiovisual en la que se puede apreciar al padre viendo una película pornográfica con su hijo, por considerar que afectaba la inviolabilidad del hogar (Art. 19 No 5 CPR). En lo pertinente la Corte de Apelaciones de Punta Arenas, en el Considerando Décimo Quinto de su sentencia declaró:"Por otra parte en cuanto a la exclusión de prueba consistente en una grabación de video, no se aprecia de qué manera se ha producido una conculcación a la garantía de inviolabilidad del hogar la que se refiere fundamentalmente al ingreso, registro o allanamiento del hogar o los espacios reservados para el trabajo o uso privado de los individuos por parte de terceros, circunstancia que no acontece en el caso de autos, toda vez que el espacio físico donde se efectuó la grabación, de acuerdo a los antecedentes, correspondía al hogar, vivienda o espacio físico que ocupa la familia que hoy se enfrenta judicialmente, razón por la cual el fundamento de la exclusión no encuentra su justificación en la garantía conculcada" 24 .

Siguiendo dicha misma línea de argumentación, anteriormente la Corte de Apelaciones de Valparaíso habría señalado en términos similares: "Que, en cuanto a que los correos electrónicos y las fotografías, habrían sido obtenidas por el demandante violando garantías constitucionales, en la especie, aunque no es señalada, la establecida en el artículo 19 No 5 de la Constitución Política de la República, en relación con el artículo 31 de la ley 19.968, necesario es tener presente que la situación planteada por el apelante, se encuentra normada por la disposición constitucional antes mencionada que establece como garantía fundamental 'La inviolabilidad del hogar y de toda forma de comunicación privada' y, además, por el inciso $2^{\circ}$ del artículo 146 del Código Penal, en efecto, la mencionada disposición legal que tipifica el delito denominado doctrinariamente como Inviolabilidad de la Correspondencia, en el inciso señalado, dispone "Esta disposición no es aplicable entre cónyuges ni a los padres, guardadores o quienes hagan sus veces, en cuanto a los papeles o cartas de sus hijos menores que se hallan bajo su dependencia. De lo anteriormente expuesto, se deduce que la parte demandante no transgredió ninguna garantía constitucional para obtener la prueba en que se fundamenta para acreditar los hechos materia de su demanda" 25 .

De la misma manera, la Corte de Apelaciones de Concepción ha entendido la excepcionalidad de la regla de exclusión en perspectiva a la libertad de prueba, señalando que la norma del artículo 31 debe ser interpretada de forma restrictiva. En el caso específico, el tribunal de alzada falló que la prueba consistente en un detalle de tráficos entre dos teléfonos de línea fija no vulnera ningún derecho fundamental, en la medida que es público

24 RODRIGGUEZ CON ARAYA (2017).

25 C.P.A.C CON N.N.M.C (2010). 
y notorio que en la documentación que se recibe para el cobro de los servicios telefónicos consta con detalle el tráfico de llamadas efectuadas ${ }^{26}$.

Por lo anteriormente tratado parece ser que, en lo meramente ilustrativo de nuestra jurisprudencia, las Cortes han tendido a comprender la regla de exclusión de prueba por vulneración de derechos fundamentales de manera restrictiva, rechazando las alegaciones a su respecto sin extenderla a criterios de difícil delimitación, sin perjuicio de no existir en lo referido a ella un análisis pormenorizado sobre la existencia de un derecho fundamental cuya vulneración deba sobreponerse a libertad de prueba vigente en el procedimiento, y en tal caso cómo se habría llevado a cabo esta conculcación. Lo anterior, con todo, supone que no se ha resuelto, o al menos no es accesible si lo estuviere, el alcance de la limitación que la eficacia de los derechos fundamentales tendría sobre la prueba ofrecida, y que, en conclusión, incumbe limitar la cantidad de prueba producida para ser rendida en la audiencia de juicio, permitiendo que se conforme o integre un material probatorio de menor calidad o fiabilidad.

\section{ETAPA DE VALORACIÓN, UTILIZACIÓN DE INFERENCIAS PROBATORIAS, ESPECIALMENTE MÁXIMAS DE LA EXPERIENCIA}

En la segunda etapa de la actividad probatoria el juez debe valorar la evidencia rendida, estableciendo el valor corroborativo que los medios de prueba tienen respecto de los enunciados sobre los hechos propuestos por las partes, es decir "en evaluar el apoyo empírico que los elementos de juicio incorporados aportan a una determinada hipótesis u otra" ${ }^{27}$. La LTF -siguiendo la línea de los procedimientos reformados (orales, públicos, en que prima la inmediación) estableció en su artículo 32 que los jueces apreciarán la prueba conforme a las reglas de la sana crítica. Así, se consagra un sistema de valoración libre, pero contenido a través del límite de la racionalidad. En ese contexto, el ensayo se detendrá específicamente en uno de los criterios que componen estos límites antes referidos, las máximas de la experiencia. Éstas serán entendidas, siguiendo a González Lagier, como inferencias probatorias epistémicas ${ }^{28}$, que los tribunales de familia deben construir al momento de la valoración de la prueba, y en cómo es que dichas inferencias debieran ser construidas desde un punto de vista lógico inductivo evitando contaminar su razonamiento con generalizaciones, prejuicios y estereotipos ${ }^{29}$.Lo anterior, resulta particularmente relevante atendido que el contenido del derecho de familia resulta esencialmente más dinámico que aquel propio del derecho privado patrimonial, por lo que la judicatura llamada a resolver conflictos de familia, en atención a dicho contenido y su permeabilidad a la realidad social

\footnotetext{
${ }^{26}$ J.A.V.A CON X.V.I.S (2012) Considerando Quinto. La sentencia señala que la exclusión de la prueba constituye una excepción al principio de libertad de prueba que consagra el art. 28 de la Ley No 19.968. Por ello, la norma de exclusión (art. 31 de la referida ley) debe ser interpretada de forma restrictiva. En el caso específico, el tribunal de alzada falló que la prueba consistente en un detalle de tráficos entre dos teléfonos de línea fija no vulnera ningún derecho fundamental, en la medida que es público y notorio que en la documentación que se recibe para el cobro de los servicios telefónicos consta con detalle el tráfico de llamadas efectuadas.

27 Ferrer (2007) p. 46.

28 Gonzalez Lagier (2014) pp. 87-88.

29 Schauer (2003), p. 15. También en Taruffo (2009) p. 156.
} 
y la de sus integrantes, se encuentra más expuesto a las precitadas falencias inferenciales en el devenir de su razonamiento inferencial.

Por inferencias probatorias epistémicas entenderemos el ejercicio lógico inferencial por el cual el sujeto llamado a decidir construye una regla en función de la observación de la reiteración de un fenómeno determinado, del cual se extrae una regla general que es capaz de cumplir determinadas funciones ${ }^{30}$. Esta regla o máxima constituye un elemento relevante para la teoría de la prueba en función de que, epistémicamente, es posible prever resultados en aplicables a casos desconocidos a partir de aquellos casos que permitieron construir la regla ${ }^{31}$. Tradicionalmente, el reconocimiento normativo a las máximas de la experiencia se ha traducido en su función limitativa de la libertad de valoración del juez ${ }^{32}$, es decir un cerrojo que impide la libertad absoluta ${ }^{33}$, o en términos de Ferrer, que la prueba "sea libertad, pero no tanto" 34 . Sin embargo, las máximas de la experiencia suelen cumplir, en lo que a la teoría de la prueba se refiere, funciones que no solo operan como límite negativo, sino que permiten, positivamente, construir o, mejor dicho, reconstruir, el relato de las partes sobre los enunciados sobre los hechos, formando parte indispensable de la cadena de inferencias que permiten tener por probado $-\mathrm{o}$ no- un enunciado fáctico en juicio. Para poder cumplir cabalmente estas funciones una máxima de la experiencia debe estar construida de forma tal que pueda erigirse sobre ella la consideración del juez respecto de los hechos. Así, señala el citado González Lagier, la máxima de la experiencia deberá cumplir con dos requisitos para poder considerarse como fiable: "a) deben estar bien fundamentadas (esto es, ser la conclusión de un argumento inductivo bien construido, que partiendo del examen de casos particulares concluya el enunciado que describe una regularidad empírica) y b) en el caso de que establezcan una regularidad probabilística (si p, entonces probablemente q), la probabilidad debe ser elevada" ${ }^{35}$. Así, en su construcción lógica el sentenciador deberá ser riguroso en su proceso de fundamentación de la generalización, evitando caer en los peligros propios que dicho ejercicio encierra, especialmente notorios en cuestiones relativas al derecho de familia.

Los referidos peligros se asilan en lo que Twining llama "stock de conocimiento" del sujeto que construye la máxima de la experiencia, basado en su experiencia personal ${ }^{36}$,

30 González Lagier (2014) pp. 87-88.

31 GonZÁLeZ Lagier (2014) pp. 87-88.

${ }^{32}$ La Jurisprudencia de la Corte Suprema ha definido, a su vez, a las máximas de la experiencia como "las que como ya se indicó, obedecen a pautas que se extraen de la observación general de la sociedad, de la cual el juez no solo forma parte, sino también se nutre. Corresponden a inferencias respaldadas por el ejemplo de conductas y hechos reiterados en el tiempo, que abstraídos de las singularidades de cada una de las situaciones concretas, permiten la confección de reglas o modelos que pueden ser tenidos por verdad, de modo que examinando sus resultados, es posible descubrir sus causa" BETANCOURT CON ORTIZ (2016).

${ }_{33}$ Así, el artículo 32 señala "Valoración de la prueba. Los jueces apreciarán la prueba de acuerdo a las reglas de la sana crítica. En consecuencia, no podrán contradecir los principios de la lógica, las máximas de la experiencia y los conocimientos científicamente afianzados".

${ }^{34}$ Ferrer (2010) p. 3.

35 González Lagier (2014) pp. 87-88.

36 Twining (2006) p. 338. 
"aglomeraciones de creencias mal definidas"37, un acervo de información que a su juicio conforma una especie de potaje, compuesto por información anecdótica, mitos y prejuicios $^{38}$. En ese contexto, este trabajo pretende hacer presente los peligros que ese stock puede aportar en la construcción inferencial del juez de familia, especialmente referido a prejuicios, estereotipos y libretos.

En primer término, se examinarán los prejuicios. Los prejuicios, señala Schauer, tratan de "creencias infundadas respecto a una persona, normalmente basadas en generalizaciones estadísticamente febles" 39 . Generalmente, los prejuicios son adecuaciones de ciertas conductas que se atribuyen respecto de grupos minoritarios o específicos de la sociedad, asociados a religión, raza o clase social ${ }^{40}$. El peligro de elaborar una cadena inferencial que parte de una premisa prejuiciada radica en su conclusión, toda vez que el prejuicio puede, y muchas veces suele, ser compartido por una parte importante de quienes componen un grupo social determinado, validando como consecuencia dicha conclusión, es decir "mal de muchos, consuelo de tontos". En relación al derecho de familia este riesgo de hace presente en casos en los que se debe decidir en función de medios de prueba que son concluyentes en sí mismos, como ocurre por ejemplo con un examen de $\mathrm{ADN}$ en una acción de reclamación. Tómese, por ejemplo, la decisión sobre un cuidado personal, en el que el juez o jueza decanta su decisión en función de la asignación prejuiciada de roles parentales, en el que se asume que la crianza a temprana edad "corresponde" a las madres y no a los padres.

Un ejemplo en nuestra jurisprudencia de familia, aplicado a cuestiones patrimoniales, es el caso en que la Corte de Apelaciones de Rancagua en el que conociendo la apelación respecto de una sentencia de primera instancia que se fijaba el monto de una compensación económica señalo que:

“TERCERO: Que sin embargo, para efectos de calcular la compensación económica no se puede tomar el total del sueldo que se dejó de percibir, pues de haberlo obtenido, las máximas de la experiencia señalan que, la mayor parte o su totalidad lo habría destinado a cubrir sus propios gastos, los que, por no haber trabajado, se entiende fueron solventados por su cónyuge, debiendo por ello realizarse una compensación imaginaria entre lo que la actora dejó de percibir y lo que su cónyuge gastó en mantenerla" ${ }^{41}$.

El considerando transcrito asume, aludiendo a un prejuicio derechamente como una verdadera máxima de la experiencia según reza su texto, que las mujeres casadas que ejercen una profesión y oficio y que generan ingresos lo gastan en "sus propios" gastos más que en el haber común del matrimonio o en el establecimiento del hogar común o los hijos, lo que, además, permite inferir que se asume, asimismo, que es el marido quien cubre, necesariamente, dichos gastos ${ }^{42}$.

\footnotetext{
37 Twining (2006) p. 338.

38 Twining (2006) p. 338. Véase También el notable trabajo de Oyarzun (2016) p. 35.

39 SChauer (2003) p. 15.

40 SCHAUer (2003) p. 15.

41 GONZALEZ CON DIAZ (2016).

42 Este ejemplo podría servir también para explicar los otros peligros aquí tratados, los libretos (scripts) y los estereotipos, según se verá más adelante.
} 
Por su parte los libretos o scripts, descritos por Taruffo, señalan que existen asignaciones ético morales referidas a cuestiones entendidas como buenas o malas en función de determinadas conductas consideradas normales o anormales. Es decir, el riesgo de que una conducta determinada sea considerada "mala" o "menos valiosa" o que la del antagonista, en función de la distancia o lejanía que tenga con lo que la generalidad considera normal, sin ninguna evidencia de patológica o científica de conflictividad ${ }^{43}$. Así, por ejemplo, se podría entender que una familia constituida por una figura materna y otra paterna es "mejor" que una monoparental, o que una familia constituida por una pareja homosexual debe ceder ante una heterosexual al momento de tomar ciertas decisiones (cuidados personales, relación directa y regular, susceptibilidad de adopción, etc. $)^{44}$. Si estos libretos preconcebidos se interponen en la elaboración de las máximas de la experiencia el resultado podría ser lógicamente inexacto y jurídicamente incorrecto.

De la misma manera, ocurriría en cuestiones patrimoniales propias de la esfera de competencia de los tribunales de familia, como pensiones de alimentos o compensaciones económicas derivadas de divorcios, en las que la asignación de roles preestablecidos prejuiciadamente o considerados más o menos valiosos podrían incidir en la construcción de la premisa fáctica por parte del sentenciador.

Finalmente, existen los estereotipos ${ }^{45}$, derivados de procesos de generalización febles y carentes de fundamentos (incluso fundados en prejuicios) por los cuales se asocian determinadas características a un grupo general de individuos ${ }^{46}$, a partir de que ciertos, algunos o determinados miembros de ese grupo participa de alguna de ellas, v.gr., "policía corrupto" "político corrupto" o asociar una determinada nacionalidad con el tráfico de drogas o un domicilio con una "facilidad delictiva" 47.

La utilización de este tipo de estereotipos en la elaboración de máximas de la experiencia podría ser peligrosa al tenor de decisiones sobre cuidado personal o relación directa y regular, e incluso respecto de la imposición de medidas de seguridad en causas de violencia intrafamiliar asociadas a estereotipos "más violentos" según clase social o nacionalidad ${ }^{48}$.

En nuestra jurisprudencia, es posible encontrar algunos ejemplos a este respecto. Así, decidiendo sobre una demanda de modificación de cuidado personal interpuesta por el padre de una menor, quien vivía en la ciudad de Concepción, en contra de la madre que residía en Ancud, el Juzgado de Familia de Concepción, fundamentando su decisión de acoger la demanda al tenor de la prueba rendida señaló en su considerando Vigésimo Primero:

\footnotetext{
43 TARUfFo (2009) p. 156.

44 De la misma manera trabajar de día a trabajar de noche o ser profesional universitario a no serlo.

45 Para una profundización reciente sobre concepto categorías y consecuencias de los estereotipos véase el trabajo de ARENa (2016).

46 Timmer (2015) p. 240.

47 TARUfFo (2009) pp. 156-157.

48 En la literatura y jurisprudencia comparada existen sensibles casos que ejemplifican estos peligros. Así, la Corte Europea de Derechos Humanos debió conocer del caso Marckx v. Belgica (1979), por el cual la justicia de Bélgica había decidido fundado en el estereotipo de madre soltera, las que "usualmente tienden a no querer hacerse cargo de su descendencia”. Citado en Timmer (2015) p. 243.
} 
“(en Chiloé) no tienen aeropuerto, ni buenos caminos, accesos expeditos, sumado a que las condiciones climáticas son difíciles para alguien que no está acostumbrado a la zona, que sus habitantes tienen una idiosincrasia especial, a diferencia de Concepción que es una urbe con todos los requerimientos e infraestructura necesarias para desarrollarse plenamente" ${ }^{29}$.

El extracto precitado expone un ejemplo claro errores en la construcción de máximas de la experiencia, así como de la utilización de un estereotipo para la cimentación de una decisión probatoria. Se trata, en este caso, de establecer -como un hecho en el procesoqué lugar de residencia se constituye como el idóneo para el mayor desarrollo espiritual y material de la menor, y en consecuencia otorgar o no el cuidado personal al padre demandante. Si bien es correcto, incluso un hecho notorio, que la isla de Chiloé vive condiciones de conectividad deficitarias, que cuenta con lugares de difícil acceso, y que tiene un clima comparativamente más extremo que el de otras zonas de Chile, la sentencia no menciona en dicho pasaje como es que ello afectaría, necesariamente, el desarrollo de la menor, o como es que una ciudad de mayor tamaño como Concepción supone un mejor lugar para su desenvolvimiento.

Lo anterior, parece emerger del acervo cultural de un juez que, situado en Concepción, construye una máxima de la experiencia inferencialmente incorrecta (el potaje del que nos habla Twining). Sin embargo, lo que más llama la atención del extracto del considerando transcrito es la referencia a los habitantes de la isla, a los que se menciona como poseedores de una "idiosincrasia especial". No nos queda claro a que podría referirse con dicho adjetivo, pero del contexto supone que existe en la isla un conjunto de costumbres o hábitos que caracterizan a la población chilota, los que además no se mencionan, y que, aunque sin decirse en forma expresa, "dificultan" el desarrollo de la menor, por oposición a lo que ocurre en una metrópoli. Esto supone la utilización del estereotipo "chilote" fundado en los prejuicios de un sujeto de hábitos urbanos en relación a lo que una isla como Chiloé puede ofrecer para el desarrollo espiritual, material y afectivo de una niña.

Otro caso llamativo, pero aún más relevante que el anterior teniendo presente que se trata de tutela cautelar en una casa de medidas de protección de menores por vulneración de sus derechos fundamentales, ocurre en la sentencia del Juzgado de Familia de Quinchao, en el que el padre de los menores solicita el cuidado personal mediante el procedimiento de urgencia ya referido, por encontrarse sus hijos expuestos a potenciales situaciones de vulneración de sus derechos fundamentales, especialmente por la conducta del conviviente de la madre. En la fundamentación de la decisión de no alterar el régimen vigente en manos de la madre el sentenciador esgrime la siguiente fundamentación al examinar la prueba rendida en la letra a) de su considerando quinto:

"Además, por su naturaleza el vínculo madre-hijo es fuerte por el hecho natural de la gestación, o sea, el hecho de llevar en su vientre al futuro vástago" 50 .

En este caso, el juez utiliza una mezcla entre estereotipo, por asignación de un rol en virtud de que la madre es mujer, y por ende "posee un vínculo fuerte por el hecho de la

\footnotetext{
49 IBARRA CON BRAVO (2017).

50 RESERVAdA (PROTECCIÓN DE MENORES) (2017).
} 
gestación”, derivando necesariamente una vinculación emocional y afectiva del solo hecho del embarazo, y además un libreto o script, pues entiende que es "bueno" o "mejor" que los hijos estén al cuidado de su madre que de su padre, sin perjuicio de que sería "incluso mejor" si pudiesen vivir juntos ${ }^{51}$.

Estas referencias, de las cuales no se pueden -ni se pretende- extraer conclusiones de carácter general, sirven para ilustrar cómo el acervo prefijado del sujeto llamado a decidir puede contaminar su decisión al valorar la prueba, mediante la feble configuración de máximas de la experiencia, las que como se ha dicho, manifiestan sus peligros en forma particularmente clara, incluso paradigmática en materias de contenido valórico, como ocurre en el caso de familia.

\section{TERCERA ETAPA, APLICACIÓN DE UN ESTÁNDAR DE PRUEBA, LA DISTANCIA ENTRE LA PROBABILIDAD PREVALECIENTE Y LA DUDA RAZONABLE}

Según se ha explicado al inicio de este trabajo, el tercer momento o etapa de la actividad probatoria es la aplicación de un estándar de prueba ${ }^{52}$. Entendido el estándar de prueba como el umbral de suficiencia probatoria que permite tener por probado un enunciado sobre los hechos ${ }^{53}$, se debe atender a su función como mecanismo de distribución de errores en el procedimiento de familia ${ }^{54}$, teniendo presente que en todo proceso el "quantum" de error que desea tolerarse marcará el límite donde se fije dicho umbral, teniendo como parámetros habituales el estándar de duda razonable del proceso penal (art. 340 CPP) y de probabilidad prevaleciente del proceso civil. Lo anterior, habida consideración de la aplicación supletoria del CPC y la naturaleza "privada" que se suele atribuir al derecho sustantivo de familia, permiten plantearse la pregunta respecto de cuál es el estándar general aplicable al procedimiento previsto en LTA, ya sea si siguiendo la natural tendencia por la alternativa a la probabilidad prevaleciente del proceso civil, o si, por el contrario, debería existir un baremo distinto en causas en que los intereses jurídicos en disputa puedan participar de una naturaleza que desborde el derecho civil patrimonial, como ocurre con diversas materias de competencia de los Juzgados de Familia y que se ilustran, paradigmáticamente, en casos de medidas de protección y violencia intrafamiliar, en los que derechamente se ejerce una potestad sancionadora por parte de la judicatura.

En general, la doctrina, y especialmente la jurisprudencia, en materia de familia no se había detenido, hasta data reciente, en el análisis del concepto de estándar de prueba, incurriendo en una sensible omisión que sido constatada habitualmente en sistemas de prueba reglada o tasada como ocurre en el caso del régimen civil bajo el actual $\mathrm{CPC}^{55}$. Se trata,

51 Considerando quinto de la sentencia letra e) señala "Que lo propio es que los hijos vivan con sus padres, así por ley humana y ley natural".

52 Ferrer (2007) p. 41, Taruffo (2008) p. 37.

53 Gascón Abellán (2005) p. 129. En términos de Haack "la cantidad de prueba que la sociedad ha señalado es requerida para poder considerar que un hecho efectivamente ha ocurrido" HAACK (2014) pp. 47-50.

54 LAUdan (2013) p. 33.

55 Fuentes (2011). 
en general de una discusión postergada o ni siquiera completamente comprendida por parte importante de los operadores del sistema, pero que, sin embargo, ha comenzado -lentamente, por cierto- a permear en el acervo jurídico cultural de la judicatura de familia.

El nudo de la discusión se devela en función de una pregunta: ¿Cuál es el estándar de prueba aplicable a las decisiones de los jueces de familia? Como se ha señalado más arriba, existe una encrucijada entre dos cuestiones que merece la pena examinar, por un lado se entiende que el estándar de prueba constituye una decisión política (valorativa) ${ }^{56}$, que debe ser determinada por el legislador en función de la aversión al riesgo de errores en un determinado tipo de proceso, en función de cómo quiera distribuir las decisiones equivocadas respecto a que un determinado enunciado sobre los hechos esté o no probado (está probado que p) y las consecuencias de dicha decisión en la aplicación del derecho según corresponda $^{57}$, configurando la decisión sobre el caso controvertido ${ }^{58}$.

Por otro lado, el derecho de familia es definido como una rama del derecho privado, pero provisto de especiales características, compuesto por un alto contenido de carácter no patrimonial $^{59}$, y que contiene la regulación de los aspectos más esenciales de las relaciones filiales y afectivas del ser humano. Adicionalmente, la jurisdicción especializada de familia ha sido dotada de competencia para el conocimiento de asuntos que, pareciera, sobrepasa este carácter puramente privado, como la violencia intrafamiliar ${ }^{60}$ y las medidas de protección por vulneración de derechos de los menores ${ }^{61}$.

En ese contexto de competencia amplia en cuanto a materias es, precisamente, donde la pregunta enunciada anteriormente toma relevancia, referida en dos hebras; la primera respecto a cuál sería el umbral de suficiencia probatoria general en materia de familia y; luego, si son equiparables desde la perspectiva del valor de los bienes jurídicos en juego y la relevancia del error diversas cuestiones de competencia de los mismos tribunales, como una compensación económica, una acción de filiación, una declaración de susceptibilidad de adopción y una condena por violencia doméstica.

A nivel jurisprudencial, vale la pena hacer presente que se ha iniciado un loable proceso de reconocimiento del tercer momento de la actividad probatoria a través de la mención expresa de estándares de prueba en las sentencias de familia, las que en data reciente han entendido que el estándar de la duda razonable debe quedar reservado al proceso penal y que es la probabilidad prevaleciente el listón a aplicar en materia de familia. En tal discurrir, sin embargo, los tribunales han señalado que inclusive en causas de naturaleza sancionatoria el estándar debe ser medio, así es posible citar a la Corte de Apelaciones de Arica que, conociendo una causa sobre VIF señaló:

\footnotetext{
56 Gascón Abellán (2005) p 130. También llama a esto una decisión de policy. Montero (2016) p. 94.

57 Gascón Abellán (2005) p 130.

58 En este sentido, con acierto Carmen Vásquez ha señalado que corresponde a la epistemología construir el estándar de prueba, pero no determinar un mínimo indispensable, "Epistemológicamente se podrá decir el cómo, pero no el cuanto" VÁsQUEZ (2013) p. 14.

59 Ramos Pazos (2010) pp. 14-17.

60 LEY No 20.066 de 2005.

61 Párrafo Primero del Título IV de la LEY No 19.968.
} 
"Cuarto: Que, la actividad probatoria en el contexto de sana crítica impone, asimismo, la necesidad de contar con algún estándar de prueba, que nos indique cuándo el juez está autorizado racionalmente para considerar que un hecho está probado, atendido el grado de confirmación que tiene en los medios de prueba. Reconocemos la inexistencia de un parámetro legal al efecto. Sin embargo, ello no impide que el juzgador fije uno que resulte adecuado al sistema probatorio impuesto. Como se trata de una decisión a favor del rigor científico (búsqueda de la verdad, de la certeza), estimamos que en la especie se puede exigir un estándar de prueba de probabilidad media, advirtiendo, no obstante, que la naturaleza especial de este tipo de conflictos, su carácter sancionatorio y las consecuencias jurídicas que conlleva la sentencia condenatoria, podría llevarnos a sostener igualmente un estándar de probabilidad alta. El estándar de probabilidad media, que se traduce bajo la fórmula "más probable que no", implica una elección entre las diversas hipótesis fácticas en juego, prefiriendo aquella que cuente con un grado relativamente más elevado de probabilidad, en razón de las pruebas que lo apoyan, debiendo, en todo caso, existir un grado mínimo necesario de confirmación probatoria, que se puede significar en una cifra equivalente, a lo menos, al 51\% de probabilidad" 62 .

Este criterio, ha sido confirmado en data reciente por la Corte de Concepción que expresó:

"Por otra parte, quien realiza la imputación [violencia intrafamiliar] debe aportar los antecedentes necesarios al proceso, en la etapa procesal correspondiente, para los efectos de formar convicción en el sentenciador -a lo menos con el estándar de preponderancia de la evidencia- acerca de la existencia de los hechos denunciados"63.

La precitada inexistencia de la consagración normativa de una regla de estándar de prueba civil, así como el criterio jurisprudencial evidenciado impone la dificultad de la primera arista de la pregunta planteada, pero que, a juicio de este trabajo, no puede contestarse sin la segunda. Así, dada las funciones del estándar de prueba parecerá razonable que éste sea fijado en atención a la materia específica más que en términos genéricos. Esta decisión podría ir, a nuestro juicio, en la siguiente línea, todas las cuestiones que versen sobre bienes jurídicos de carácter patrimonial que deban ser conocidos en los Tribunales de Familia son asimilables a contiendas civiles en que lo que se discute con intereses privados, generalmente disponibles y renunciables por las partes y a las que, doctrinariamente, se les ha fijado como estándar de prueba la probabilidad prevaleciente.

Sin embargo, cuestiones de orden público y carácter indisponibles, como las que versan sobre el estado civil en ciertos casos, como las susceptibilidades de adopción; y especialmente aquellas que establecen sanciones de carácter quasi-punitivo, parecen presentar la necesidad de revisar si la distribución de errores no merece un grado de suficiencia probatoria mayor. Este grado más elevando no podría equipararse al nivel de la duda razonable penal, en atención a que la afectación de los bienes jurídicos de libertad individual por parte del Estado no está en juego, pero sí plantea la posibilidad de un umbral intermedio.

62 DENUNCIANTE CON N.F.A (2010).

63 Contreras con Figueroa (2017). 
A este respecto, en el derecho comparado se ha establecido que es posible utilizar estándares intermedios, como la prueba clara y convincente ${ }^{64}$, que sirvan de línea medianera entre el estándar civil y la duda razonable penal. Estos estándares intermedios suponen un umbral más alto que la simple alocución "más probable que no", pero no llegan a la altísima vara de la duda razonable, permitiendo ponderar, como señala Larroucau, "la igual consideración y el debido respeto por los intereses en juego" ${ }^{65}$, pero que dados los intereses litigados "justifica acentuar la dirección de los riesgos hacia el actor" ${ }^{66}$. Sin perjuicio de la problemática que plantea traducir un estándar de prueba a números, constituye una herramienta metodológicamente útil expresar este baremo intermedio entre el 0,5 y el 1 en un 0,75, como se ha hecho en el caso de la jurisprudencia de Estados Unidos ${ }^{67}$.

Al otro lado del Atlántico la jurisprudencia de las cortes inglesas ha señalado que, en realidad, no corresponde mover formalmente la vara de suficiencia probatoria civil en casos complejos en que se trata de cuestiones puramente civiles, ni aún en el caso de que se realicen alegaciones referidas a infracciones penales ${ }^{68}$. Sin embargo, la jurisprudencia ha encontrado grandes problemas en lograr asentar un estándar de prueba específico para cuestiones de familia, tomando siempre en consideración la gravedad de las consecuencias del error $^{69}$. Así, en casos de divorcio por adulterio, en que la consecuencia era la destrucción de la presunción de filiación respecto del marido, la relevancia de la indeterminación de la filiación de los hijos hizo pensar, habiendo sentencias en tal sentido ${ }^{70}$, que el adulterio debía probarse en un umbral mayor, incluso al extremo de la regla penal, sin perjuicio de que esta tendencia haya ido hoy desapareciendo ${ }^{71}$. De la misma manera, en casos sobre filiación (reclamación) se ha dicho que, dado el peso del asunto, se requiere un estándar al menos levemente mayor que el del balance de las probabilidades ${ }^{72}$.

Siguiendo las tendencias comparadas mediante una decisión legislativa se puede distribuir asimétricamente el riesgo de error en el proceso según sea la intención del legislador de tutelar ciertos errores por sobre otros, alejando el contenido no patrimonial del derecho de familia del paradigma de igualdad formal de la litigación civil, privilegiando la posición de ciertos sujetos en relación al objeto de cada disputa según materia.

\section{RELEVANCIA ACTUAL DE LA EXPOSICIÓN Y REFLEXIONES FINALES}

Durante la exposición que antecede se ha intentado, con una mesurada intención de efectividad, por cierto, utilizar la construcción doctrinaria de la teoría de la prueba relativa

\footnotetext{
64 "Clear and convincing proof, o clear, and satisfactory evidence". FUENTES (2011) p. 192.

65 Larroucau (2012).

66 Larroucau (2012).

67 Larroucau (2012).

68 ZuCKerMan (2013) p. 1021.

69 Keane y McKeown (2014) pp. 112-113.

70 Por ejemplo, Ginesi v. Ginesi [1948] P 179, CA y Preston-Jones v Preston-Jones [1951] AC 391.

71 Keane y Mckeown (2014) p. 113.

${ }^{72} W v K$ [1988] Law Fam 64 y Serio $v$ Serio [1983] 13 Fam Law 255. Ambos citados por Keane y MCKEOWN (2014) p. 115.
} 
a los momentos o etapas de la actividad probatoria para ilustrar ciertas cuestiones que representan desafíos en la LTA. Este entramado normativo, prisma procesal del derecho de familia, permite, al igual que otros procedimientos reformados, la diferenciación de las etapas de inclusión, valoración y estándar con una notoria ventaja por sobre el CPC, aunque sin la claridad que, por ejemplo, refleja respecto de ellos el CPP. Con ello, hemos presentado algunos problemas que cada etapa probatoria presenta de cara a la praxis judicial, en especial atención al tratamiento de bienes jurídicos y cuestiones de orden público, de gran relevancia para el individuo y que incluso alcanzan la punición y/o restricción de derechos de los justiciables. Así, se han expresado dudas respecto a las normas que regulan la exclusión de prueba, a las máximas de la experiencia y los riesgos de esquemas preconcebidos en el acervo cultural del juez, y finalmente a la pertinencia de la aplicación de un (determinado) estándar de prueba en la decisión de familia. Cada una de estas temáticas se presenta con mayor énfasis de cara a una judicatura de familia que desde su creación ha enfrentado -y sigue enfrentando- algo que podría denominarse como "hipertrofia competencial", es decir, una dotación de competencia cada vez mayor, que redunda en que cada vez más asuntos caen bajo la potestad jurisdiccional de los jueces de familia.

Lo anterior nos obliga a pensar en la reciente atribución de competencia a los jueces de familia para la resolución de dos cuestiones que han connotado enorme debate social, tales como la Ley No 21.030 sobre Interrupción Voluntaria del Embarazo en tres causales, que en su artículo primero regula el procedimiento judicial para la obtención de la autorización sustitutiva de la voluntad del representante legal en el caso de la interrupción voluntaria del embarazo de una menor de 14 años $^{73}$; y, en segundo término lo que ocurre con la Ley de Identidad de Género, Ley No 21.120, en la que el art. 13 entrega a los Tribunales de Familia la competencia para conocer las solicitudes de mayores de 14 años y menores de 18, así como el artículo 18, respecto de los mayores de 18 que tengan vínculo matrimonial vigente $7^{74}$. De la misma manera, no sería extraño que futuros proyectos como la adopción homoparental e incluso la eutanasia sugieran la misma suerte, introduciendo de alguna manera la tutela jurisdiccional de familia en dichas temáticas.

De esta manera, lo recién expresado en esta exposición ha intentado evidenciar, puede hacer aún más evidentes los problemas referidos, especialmente aquellos relacionados al rol del juez de cara al material probatorio presentado ante él o ella y en el que se pueden acentuar problemas para la exclusión de pruebas entre padres e hijos o familiares muy cercanos, así como la proyección de prejuicios o estereotipos del propio sentenciador respecto a sus propias creencias en la valoración a través de la construcción de máximas de

\footnotetext{
73 Art. $1^{\circ}$ LEY No 21.030 de 2017 "La autorización judicial sustitutiva regulada en los incisos anteriores será solicitada al juez con competencia en materia de familia del lugar donde se encuentre la menor de 14 años o la mujer judicialmente declarada interdicta por causa de demencia. El procedimiento será reservado y no será admitida oposición alguna de terceros distintos del representante legal que hubiere denegado la autorización. La resolución será apelable y se tramitará según lo establecido en el artículo 69, inciso quinto, del Código Orgánico de Tribunales".

74 Art. 13 LEY No 21.120 de 2018 "DEL TRIBUNAL COMPETENTE Y SUPLETORIEDAD. En caso de solicitudes de personas mayores de catorce y menores de dieciocho años, será competente para conocer la solicitud el tribunal con competencia en materias de familia correspondiente al domicilio del solicitante [...]”.
} 
la experiencia. Para finalizar, es de esperar que los riesgos aquí expuestos de forma teórica no encuentren mayor correlato en estrados, permitiendo una aplicación adecuada del principio de libertad de prueba en cada una de sus etapas y estadios procesales en aras de una decisión dotada de más racionalidad y menos arbitrariedad, especialmente en materias de gran importancia para la configuración del individuo y su entorno, y que, asimismo, suponen cuestiones socialmente relevantes. Lo anterior se facilita, desde luego, con el aumento del interés en temáticas relativas a la teoría de la prueba, su profundización y tratamiento en instancias internacionales como la presente, y que pavimentan el camino hacia un mejor entendimiento del derecho probatorio por parte de todos quienes conformamos la cultura jurídica interna.

\section{BIBLIOGRAFÍA CITADA}

Arena, Federico (2016): "Los estereotipos normativos en la decisión judicial. Una exploración conceptual", Revista de Derecho Universidad Austral, año 29, vol. 1: pp. 51-75.

Bayon, Juan Carlos (2010): "Epistemología, Moral y prueba de los Hechos. Hacia un Enfoque no Benthamiano”, Revista Mario Alfaro D’ Filippo, año 2, No 4: pp. 6-30.

Bentham, Jeremy (2001): Tratado de las Pruebas Judiciales (Granada, Editorial Comares).

Ferrer, Jordi (2005): Prueba y verdad en el derecho (Madrid, Marcial Pons).

Correa, Claudio (2016): "La prueba ilícita de los particulares: de cargo y de descargo", Revista Política criminal, año 21, vol. 11: pp. 104-139.

FERrer, Jordi (2007): La valoración racional de la prueba (Madrid, Marcial Pons).

FERRER, Jordi (2010): "La prueba es libertad, pero no tanto. Una teoría de la prueba cuasibenthamiana", en: ACCATino, Daniela (coord.), Formación y valoración de la prueba en el proceso penal (Santiago, AbeledoPerrot, LegalPublishing) pp. 3-19.

Fuentes, Claudio (2011) "Consideraciones en torno a la idea del estándar de convicción en el proceso civil” en: LetURIA, Francisco (edit) Justicia civil y comercial: Una reforma ¿cercana? (Santiago, Ediciones LyD) pp. 173-205.

Fuentes, Claudio (2011): "La persistencia de la prueba legal en la judicatura de familia", Revista de derecho (Coquimbo), año 18, vol. 1: pp. 119-145.

Gascón Abellán, Marina (2005): "Sobre la posibilidad de formular estándares de prueba objetivos", Doxa: Cuadernos de Filosofía del Derecho, año 28: pp. 127-139.

Glover, Richard (2017): Murphy on Evidence (Oxford, Oxford University Press).

Gonzalez Lagier, Alejandro (2014): "Presunción de inocencia, verdad y objetividad" en Garcia, Juan y Bonorino, Pablo. (Coords.) Prueba y razonamiento probatorio en Derecho, debates sobre abducción (Madrid, Editorial Comares) pp. 85-116.

Jara, Francisco y Jara, Camilo (2014): "La Prueba Ilícita en materia de Familia", Revista Colegio de Ayudantes Derecho UC. Disponible en: https://www.coaduc.cl/revistacoaduc/ volumen-1-2014/. Fecha de consulta: 30 de noviembre de 2018.

Haack, Susan (2014): Evidence Matters: Science, Proof, And Truth In The Law (Cambridge, Cambridge Univerity Press).

Keane, Adrian y Mckeown, Paul (2014): The modern law of evidence. (Oxford, Oxford University Press). 
Larroucau, Jorge (2012): "Hacia un estándar de prueba civil", Revista chilena de Derecho, año 39, vol. 3: pp. 783-808.

LAUdAn, Larry (2013): Verdad, error y proceso penal (Madrid, Editorial Marcial Pons).

Montero, Raúl (2016): Necesidad de establecer un estándar de prueba en el nuevo proceso civil chileno (Santiago, Librotecnia).

Oyarzun, Felipe (2016): Aplicación de las Máximas De La Experiencia en un Modelo de Valoración Racional de La Prueba. Memoria de prueba para optar al grado de Licenciado en Ciencias Jurídicas y Sociales por la Universidad de Chile.

Ramos Pazos, René (2010): Derecho de Familia, Tomo I (Santiago, Editorial Jurídica de Chile).

Roberts, Paul y Zuckerman, Adrian (2010): Criminal Evidence (Oxford, Oxford University Press).

Schauer, Frederick (2003): Profiles, Probabilities and Stereotypes. (Harvard, Harvard University Press).

Stein, Alex (2005): Foundations of Evidence Law (Nueva York, Oxford University Press).

TARuffo, Michele (2008): La prueba (Madrid, Marcial Pons).

Taruffo, Michele (2009): Páginas sobre justicia civil (Madrid, Marcial Pons).

Timmer, Alexandra (2015): "Judging Stereotypes: What the European Court of Human

Rights Can Borrow from American and Canadian Equal Protection Law”, American Journal of Comparative Law, año 63, vol. 1: pp. 239-284.

Twining, William (2006): Rethinking Evidence. Exploratory Essays. (Cambridge, Cambridge University Press).

Valenzuela, Jonatan (2017): Hechos, pena y proceso (Santiago, Editorial Rubicon).

VÁzQuez, Carmen (2013): Estándares de Prueba y Prueba Cientifica: Ensayos de Epistemología Jurídica (Madrid, Marcial Pons).

ZuCKerman, Adrian (2013): Zuckerman on civil process, principles and practice (Londres, Sweet and Maxwell).

\section{NORMAS CITADAS}

LEY No 19.968 (30/8/2004), Crea los Tribunales de Familia.

LEY No 20.066 (7/10/2005), Establece Ley de Violencia Intrafamiliar.

LEY No 21.030 (23/9/2017), Regula la despenalización de la interrupción voluntaria del embarazo en tres causales.

LEY No 21.120 (10/12/2018), Ley sobre identidad de género.

Código Procesal Penal (12/10/2000).

Código de Procedimiento Civil (30/8/1902).

\section{JURISPRUDENCIA CITADA}

Contreras con Figueroa (2017): Corte de Apelaciones de Concepción, 4 septiembre 2017, (Violencia Intrafamiliar) Rol No 405-2017. 
IBARRA CON BRAVO (2017): Juzgado de Familia de Concepción, 25 de abril de 2017 (acción de modificación de cuidado personal). RIT 125-2016.

Reservada (PRotecCión DE menores) (2017): Juzgado de Familia de Quinchao, 22 de marzo de 2017. RIT: P-74-2016.

Rodríguez con Araya (2017): Corte de Apelaciones de Punta Arenas, 11 de mayo de 2017, (Protección), Rol No 33-2017.

Betancourt CON ORTiz (2016): Corte Suprema, 25 de julio de 2016, (Alimentos). Thomson and Reuters Identificador CL/JUR/5198/2016. Fecha de consulta 30 de noviembre de 2018.

GONZÁLEZ CON Díaz (2016): Corte de Apelaciones de Rancagua, 11 de noviembre de 2016, (compensación económica), identificador Thomson and Reuters CL/JUR/8075/2016, fecha de consulta 28 de octubre de 2018.

J.A.V.A CON X.V.I.S (2012): Corte de Apelaciones de Concepción, 10 de diciembre de 2012, (Divorcio sanción) identificador Vlex VLEX-412702838. Fecha de consulta 25 de octubre de 2018.

C.P.A.C CON N.N.M.C (2010): Corte de Apelaciones de Valparaíso, 22 de diciembre de 2010, (Divorcio), Rol No 504-2010.

DENUNCIANTE CON N.F.A (2010) Corte de Apelaciones de Arica, 23 agosto 2010, (Violencia Intrafamiliar) Identificador Vlex: VLEX-226580907. Fecha de consulta 30 de noviembre de 2018.

A. con S. (2015): Corte de Apelaciones de Valparaíso, 28 de diciembre de 2015 (Divorcio por culpa) Rol 758-2015.

Betanzo CON Fuentes (2011): Corte de Apelaciones de San Miguel, 24 de enero de 2011 (Alimentos) Rol 801-2010. 